\title{
Article \\ Trends in Frailty and Use of Evidence-Based Pharmacotherapy for Heart Failure in Australian Hospitalised Patients: An Observational Study
}

\author{
Yogesh Sharma ${ }^{1,2, * \mathbb{D}}$, Chris Horwood ${ }^{3}$, Paul Hakendorf ${ }^{3}$ and Campbell Thompson ${ }^{4}$ (D) \\ 1 Department of General Medicine, Division of Medicine, Cardiac \& Critical Care, Flinders Medical Centre, \\ Adelaide 5042, Australia \\ 2 College of Medicine and Public Health, Flinders University, Adelaide 5042, Australia \\ 3 Department of Clinical Epidemiology, Flinders Medical Centre, Adelaide 5042, Australia; \\ Chris.Horwood@sa.gov.au (C.H.); Paul.Hakendorf@sa.gov.au (P.H.) \\ 4 Discipline of Medicine, The University of Adelaide, Adelaide 5005, Australia; \\ Campbell.Thompson@adelaide.edu.au \\ * Correspondence: Yogesh.Sharma@sa.gov.au
}

Citation: Sharma, Y.; Horwood, C.; Hakendorf, P.; Thompson, C. Trends in Frailty and Use of Evidence-Based Pharmacotherapy for Heart Failure in Australian Hospitalised Patients: An Observational Study. J. Clin. Med. 2021, 10, 5780. https://doi.org/ $10.3390 /$ jcm 10245780

Academic Editor: Andrea Frustaci

Received: 14 November 2021

Accepted: 9 December 2021

Published: 10 December 2021

Publisher's Note: MDPI stays neutral with regard to jurisdictional claims in published maps and institutional affiliations.

Copyright: (c) 2021 by the authors. Licensee MDPI, Basel, Switzerland. This article is an open access article distributed under the terms and conditions of the Creative Commons Attribution (CC BY) license (https:// creativecommons.org/licenses/by/ $4.0 /)$.
Abstract: Frailty increases morbidity and mortality in heart failure (HF) patients. Current riskadjustment models do not include frailty-status and the relationship between frailty and pharmacotherapy is unclear. This study explored trends in frailty over time and its relationship with prescription of heart failure specific pharmacotherapy in hospitalised HF patients. We used the Hospital Frailty Risk Score (HFRS) to determine frailty status of patients $\geq 18$ years admitted between 2015-2019 at two tertiary hospitals in Australia. Patients with an HFRS $\geq 5$ were classified as frail. In the 3706 patients with a mean (SD) age of 76.1 (14.4) years, $876(23.6 \%)$ were classified as frail. HFRS was weakly correlated with age $(\mathrm{r}=0.16)$ and Charlson-index $(\mathrm{r}=0.35)$ (both $p$ values $<0.001)$. Whilst frailty was more common in older HF patients ( $28.9 \%$ of patients $\geq 80$ years), $15.1 \%$ of patients $\leq 65$ years of age were also found to be frail. The proportion of frail patients increased from $19.4 \%$ in 2015 to $29.2 \%$ in 2019 despite no significant change in age during this period. The proportion of patients who received heart failure specific pharmacotherapy decreased from $86.7 \%$ in 2015 to $82.9 \%$ in 2019 ( $p$ value $=0.03$ ) and frail patients were significantly less likely to be prescribed HF specific pharmacotherapy than non-frail patients $(77.4 \%$ vs. $85.9 \%, p<0.001)$.

Keywords: heart failure; frailty; heart failure specific medications; hospitalised patients; mortality; readmissions

\section{Introduction}

Frailty is an important prognostic factor among patients with heart failure because it increases both morbidity and mortality [1]. The current risk adjustment models determining prognosis in heart failure, however, does not incorporate frailty [2,3]. This may be because frailty assessment requires face-to-face interaction and some frailty assessment tools require special physical measurements, such as hand grip strength or a measure of gait speed [4,5]. The Hospital Frailty Assessment Tool (HFRS) has recently been developed in the UK, and can be generated based on routinely available hospital administrative data [6]. Although this tool was originally developed for individuals aged 75 years or older, it has been validated and is generalisable in different age groups and health care settings $[7,8]$.

A number of studies [9-12] have demonstrated that despite benefits of targeted therapy among heart failure patients, the proportion of patients receiving such therapy has changed little in the last few years. Evidence $[9,10]$ suggests that older patients, those with more frequent hospitalisations, renal insufficiency, lower blood pressure and a greater severity of heart failure (NYHA class 3 and 4) are less likely to receive any (let alone achieve targeted dose of) heart failure specific medications. It is often assumed that the reason 
for non-prescription or non-achievement of the targeted dose of heart failure specific medications in these patients is due to an increasing age or the higher risk of frailty in these patients [13]. Recent studies [3,10] investigating prescription practices among heart failure patients have emphasised the need for frailty assessment. Thus far, only one study [3] has investigated the trends in frequency of frailty among hospitalised heart failure patients. To our knowledge, there has been no study in the Australian health care settings which has investigated the trends in frailty over time or the prescription of heart failure specific pharmacotherapy with regards to each patient's frailty status. This study therefore was designed to examine the prevalence and the influence of frailty over time in a cohort of patients admitted to hospital with a diagnosis of heart failure. In particular, this study examined the outcomes of admissions of these patients and the association between frailty and prescription of heart failure medications.

\section{Materials and Methods}

This retrospective study included data of all patients $\geq 18$ years of age who were hospitalised with heart failure over a period of five years at two tertiary teaching hospitals, Flinders Medical Centre (FMC) and Royal Adelaide Hospital (RAH) in Adelaide, Australia. The ethical approval for this study was granted by the Southern Adelaide Human Research Ethics Committee. We identified all adult hospital admissions, between 1 January 2015 and 31 December 2019, with a primary diagnosis of heart failure by using the International Classification of Diseases Tenth Revision Australian Modification (ICD-10-AM) code 150, which has been previously used to define heart failure [14]. In cases where patients had multiple presentations for heart failure during the study period, then only the first admission was included.

The HFRS were calculated according to the criteria defined by Gilbert et al. [6]. HFRS is based upon administrative data by allocating point values for any of 109 select ICD codes as defined in the original publication. These codes include diagnoses such as falls, osteoporosis, spinal compression fractures, blindness, skin ulcers, delirium/dementia, Parkinson's disease, urinary incontinence, urinary tract infections, disorders of electrolytes, drugs/alcohol abuse and sequelae of stroke such as hemiplegia and dysphagia. None of the ICD-10 codes used for the generation of the HFRS score is for heart failure, atrial fibrillation or coronary artery disease (CAD). Higher HFRS scores indicate a greater severity of frailty, and these scores can further be categorised into 3 groups: $>15$ (high risk), 5-15 (intermediate risk) and $<5$ (low risk). For this study, we classified patients with an HFRS score $\geq 5$ as frail and those with HFRS scores of $<5$ as non-frail as has been conducted in previous studies $[3,6]$. We examined trends in frailty status at admission by determining the median HFRS scores and the proportion of frail patients according to their age group. Trends in frailty status, as determined by the HFRS over 5 years, were examined using linear regression on corresponding year for means of continuous variables, quantile regression on corresponding year for medians of continuous variables and the use of the CochranArmitage trend test [15] for proportion of frail patients. We used Pearson correlation to test association between the HFRS and age, Charlson comorbidity index (CCI) and length of hospital stay (LOS), and Spearman's correlation was used for heart failure specific medications (beta blockers, angiotensin converting enzyme inhibitors (ACEi)/angiotensin receptor blockers (ARBs) and mineralocorticoid receptor antagonists (MRA).

The outcomes examined included: LOS, in hospital mortality, 30-day mortality (including in hospital mortality), 180-day mortality, 30-day readmissions and placement in a nursing home. We used multilevel mixed effects Poisson and logistic regression models to determine association between the outcome variables and frailty after adjustment for age, sex, CCI, creatinine, troponin, brain natriuretic peptide (BNP), albumin and c-reactive protein (CRP) levels. We also determined whether inclusion of the HFRS provides any additional information above and beyond that provided by age, sex, CCI and LACE scores when predicting clinical outcomes. The LACE score [16] gives a maximum of 19 points for hospital LOS (L), acuity of presentation (A), Charlson index (C) and emergency room 
visits in the previous 6 months (E) and has been validated for predicting readmissions or death within 30 days of hospital discharge. In addition, we also analysed outcomes in three predefined age categories ( $<65$ years, $65-79$ years and $\geq 80$ years). The trends in outcomes over time were also analysed according to the three frailty categories.

\section{Results}

There were 5191 admissions with heart failure between 1 January 2015 and December 2019. After omitting multiple admissions, 3706 patients remained in the dataset (Figure 1). The mean age was 76.1 (14.4) years, range 19-105 years and 51.7\% were males. The mean (SD) HFRS score was 3.2 (3.8) and 876 (23.6\%) patients were found to be frail (HFRS $\geq 5$ ). HFRS defined frailty was more likely to be associated with an older age, male sex, a higher CCI, increased creatinine and CRP levels but with lower haemoglobin and albumin levels (Table 1). Frailty was, however, not found to be associated with BNP or troponin levels. The HFRS was only weakly correlated with age $(\mathrm{r}=0.16, p<0.001)$ and CCI $(\mathrm{r}=0.35, p<0.001)$ in these patients.

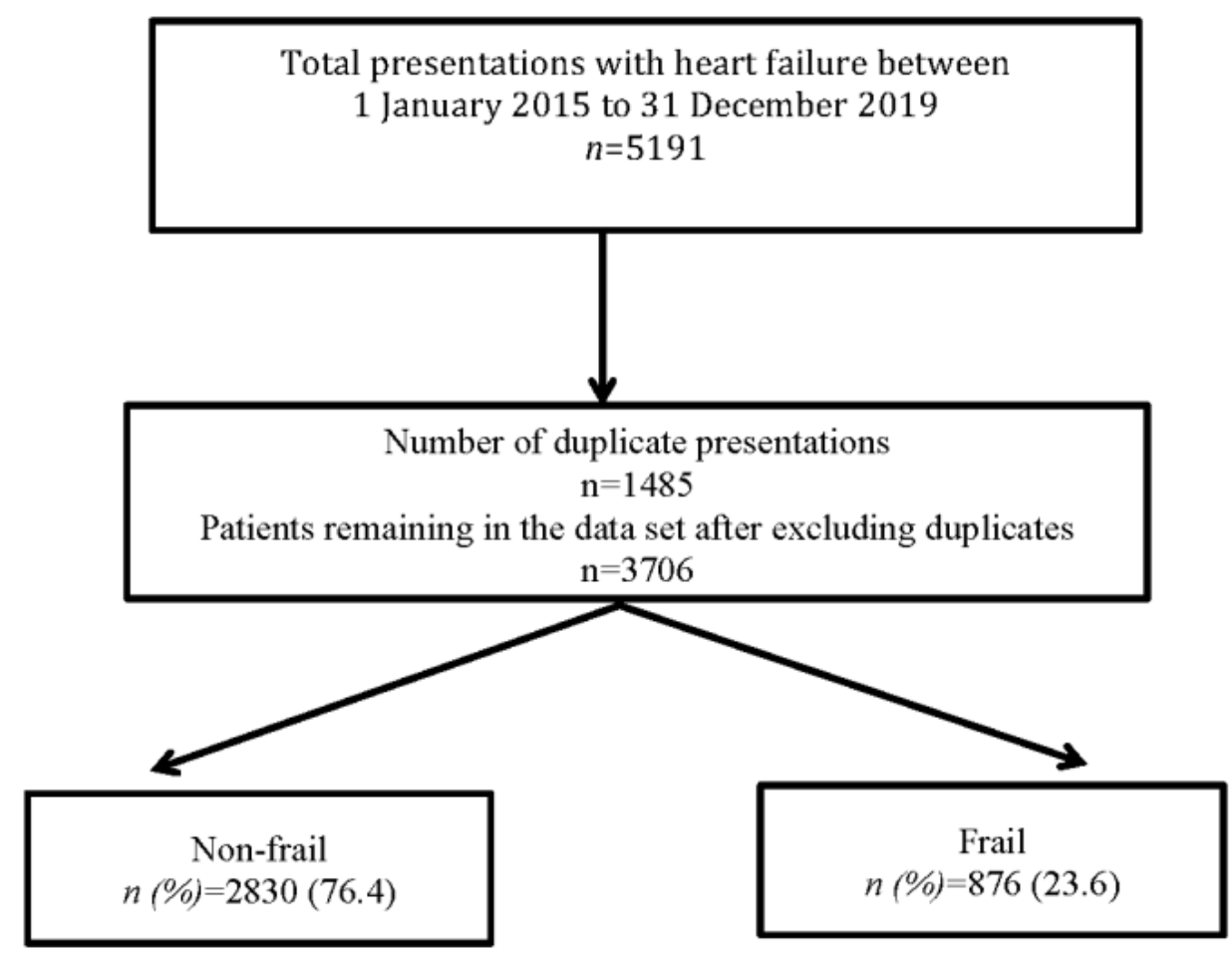

Figure 1. Study flow diagram.

The median (IQR) HFRS increased from 1.8 (0 to 4.1) in 2015 to 2.6 (0 to 5.5) in 2019 (trend $p$ value $<0.001)$ although there was no significant change in median (IQR) age during this period $80.0(69,86)$ years in 2015 vs. $80.0(68,86)$ years in $2019(p=0.092)$. The proportion of patients who were classified as frail according to the HFRS increased from $19.4 \%$ in 2015 to $29.2 \%$ in 2019 if all age groups were combined and from $21.3 \%$ to $31.3 \%$ in those over the age of 65 years (Figures 2 and 3). As expected, frailty was more common in older patients $(28.9 \%$ of patients 80 years or older and $20.2 \%$ of patients between 65-79 years); however, even in patients who were younger than 65 years, a significant proportion $(15.1 \%$ ) of inpatients with heart failure met the HFRS case definition for frailty and the proportion of patients with frailty increased in all age groups between 2015 to 2019 $(p<0.001)$ (Figures 2 and 3). 
Table 1. Baseline characteristics and use of heart failure specific medications according to frailty status.

\begin{tabular}{|c|c|c|c|c|}
\hline Variable & Low Risk HFRS & Intermediate Risk HFRS & High Risk HFRS & $p$ Value \\
\hline & $($ HFRS $<5)$ & (HFRS 5-15) & (HFRS > 15) & \\
\hline & $n=2830$ & $n=817$ & $n=59$ & \\
\hline Age years mean (SD) & $75.2(14.4)$ & $79.4(12.3)$ & $82.5(11.1)$ & $<0.001$ \\
\hline \multicolumn{5}{|l|}{ Age group $n(\%)$} \\
\hline$<65$ years & $598(21.1)$ & $101(12.4)$ & $5(8.5)$ & $<0.001$ \\
\hline $65-79$ years & $902(31.9)$ & $219(26.8)$ & $9(15.2)$ & \\
\hline$\geq 80$ years & $1330(47.0)$ & $497(60.8))$ & $45(76.3)$ & \\
\hline Sex male $n(\%)$ & $1463(51.7)$ & $414(50.7)$ & $40(67.8)$ & 0.039 \\
\hline HFRS mean (SD) & $1.5(1.5)$ & $8.0(2.5)$ & $18.0(2.7)$ & $<0.001$ \\
\hline CCI mean (SD) & $2.1(1.5)$ & $3.3(1.9)$ & $3.9(1.8)$ & $<0.001$ \\
\hline Creatinine mmol/L mean (SD) & $114.6(67.4)$ & $155.7(92.5)$ & $172.1(92.3)$ & $<0.001$ \\
\hline Haemoglobin g/L mean (SD) & $124.2(20.8)$ & $118.1(22.2)$ & $118.5(23.1)$ & $<0.001$ \\
\hline BNP mean ng/L (SD) & $1050.9(1514.8)$ & $2040.0(2076.1)$ & $1020.5(1030.7)$ & 0.468 \\
\hline Troponins ng/L mean (SD) & $1.7(31.3)$ & $0.6(7.8)$ & $0.7(3.9)$ & 0.567 \\
\hline CRP mg/L mean (SD) & $21.6(32.0)$ & $33.1(45.3)$ & $35.8(41.8)$ & $<0.001$ \\
\hline Albumin g/L mean (SD) & $34.0(4.9)$ & $32.3(5.5)$ & $31.6(3.9)$ & $<0.001$ \\
\hline MUST scores & $0.5(0.9)$ & $0.7(1.1)$ & $0.8(1.1)$ & 0.017 \\
\hline Beta blockers $n(\%)$ & $1813(64.1)$ & $460(56.3)$ & $23(38.9)$ & $<0.001$ \\
\hline ACE inhibitors $n(\%)$ & $1295(45.8)$ & $287(35.1)$ & $17(28.8)$ & $<0.001$ \\
\hline $\mathrm{ARB} n(\%)$ & $402(14.2)$ & $107(13.1)$ & $8(13.6)$ & 0.720 \\
\hline MRA $n(\%)$ & $1350(40.6)$ & $301(36.9)$ & $19(32.2)$ & 0.005 \\
\hline Sacubitril/Valsartan $n(\%)$ & $53(1.9)$ & $11(1.4)$ & 0 & 0.352 \\
\hline Aspirin $n(\%)$ & $1084(38.3)$ & $265(32.4)$ & $19(32.2)$ & 0.007 \\
\hline Warfarin $n(\%)$ & $501(17.7)$ & $192(23.3)$ & $12(20.3)$ & 0.001 \\
\hline DOACs $n(\%)$ & $648(22.9)$ & $171(20.9)$ & $8(13.6)$ & 0.131 \\
\hline Digoxin $n(\%)$ & $398(14.1)$ & $136(16.6)$ & $11(18.6)$ & 0.128 \\
\hline Statins $n(\%)$ & 1459 (51.6) & $373(45.7)$ & $17(28.8)$ & 0.001 \\
\hline SGLT2 inhibitors $n(\%)$ & $53(1.9)$ & $17(2.1)$ & $1(1.7)$ & 0.922 \\
\hline Ivabradine $n(\%)$ & $68(2.4)$ & $17(2.1)$ & $1(1.7)$ & 0.821 \\
\hline
\end{tabular}

HFRS, hospital frailty risk score; SD, standard deviation; CCI, Charlson comorbidity index; BNP, brain natriuretic peptide; CRP, C-reactive protein; MUST, malnutrition universal screening tool; ACE, angiotensin converting enzyme; ARB, angiotensin receptor blockers; MRA, mineralocorticoid receptor antagonist; DOACs; direct oral anticoagulants; SGLT2, sodium glucose transport protein 2.

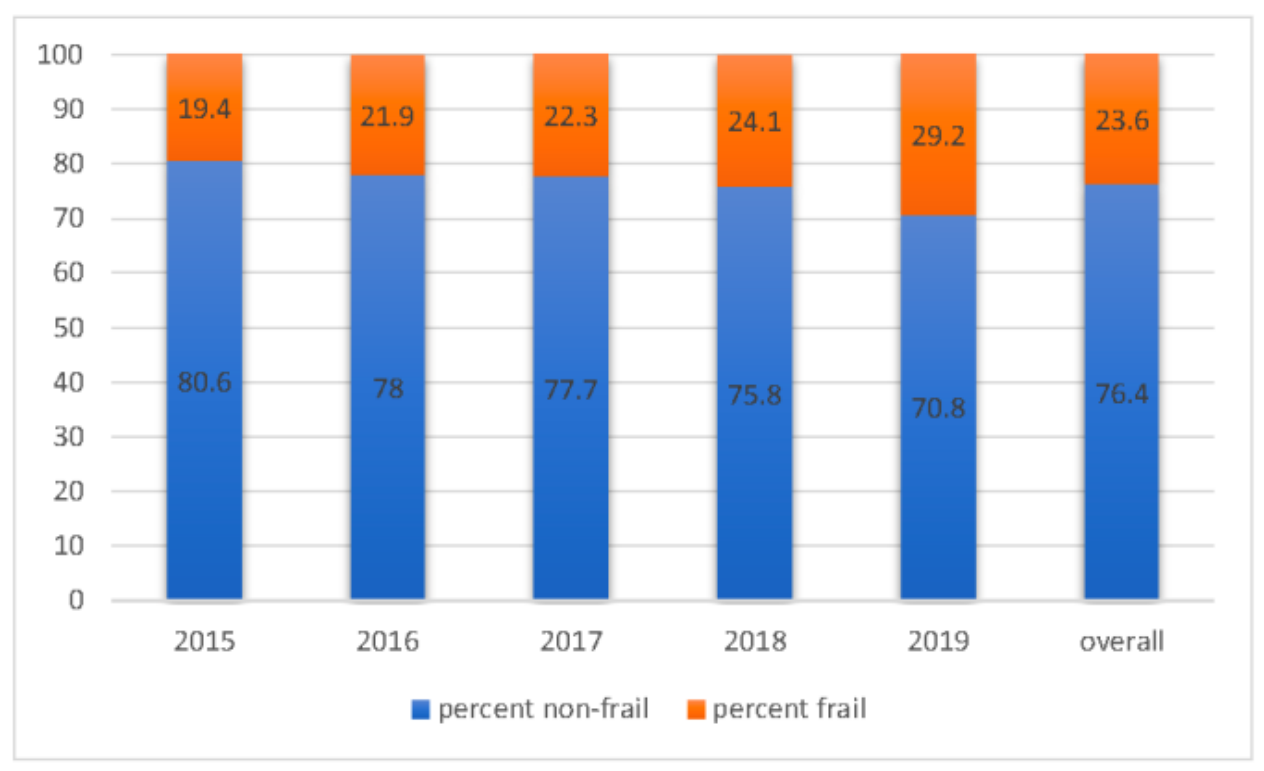

Figure 2. Temporal trends in frailty as defined by the Hospital Frailty Risk Score (HFRS) among heart failure patients from 2015 to 2019. 


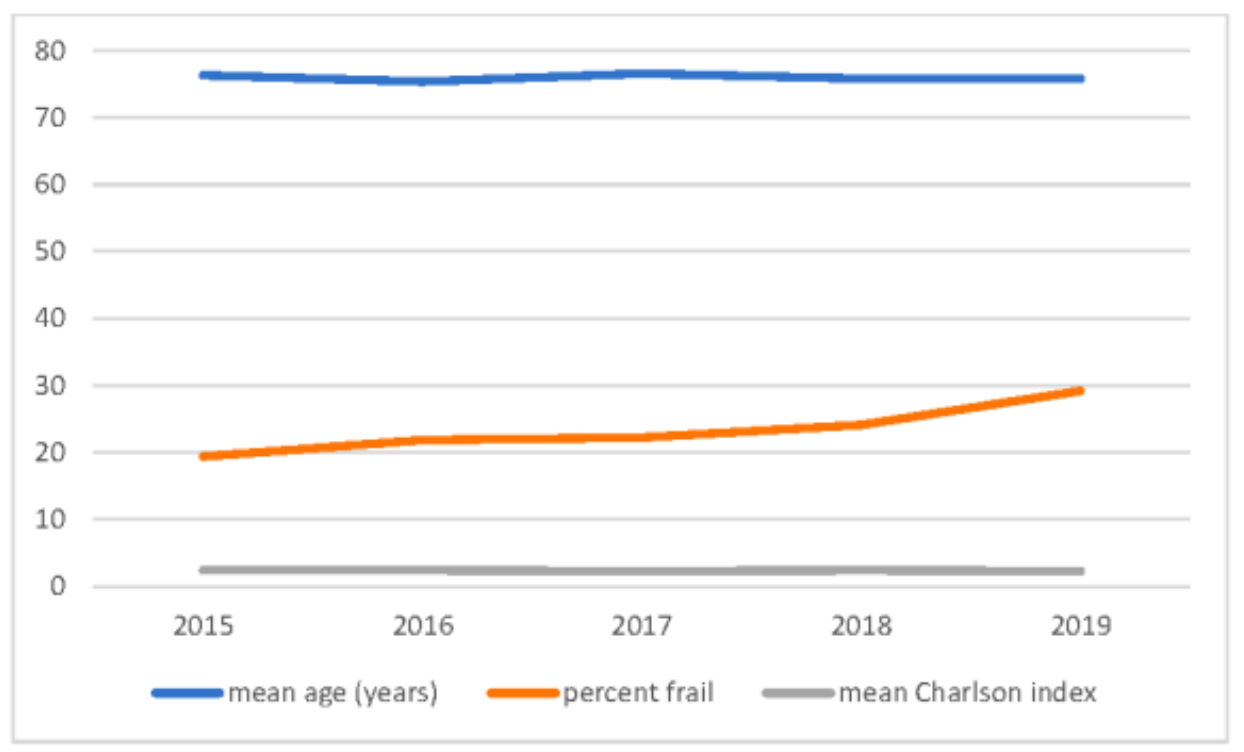

Figure 3. Graph showing trends in age, frailty status and Charlson comorbidity index over the duration of the study.

The LOS was significantly prolonged among patients who were identified as frail according to the HFRS when compared to the non-frail group (Table 2). LOS was moderately correlated with HFRS $(r=0.44, p<0.001)$. The risks of 30-day and 180-day mortality were both significantly higher in frail patients and in all three age groups (Table 2). Frailty was associated with worse clinical outcomes in terms of LOS, 30-day and 180-day mortality in both younger patients ( $<65$ years) and older patients ( $\geq 65$ years) when compared to those who were classified as non-frail $(p<0.05)$. When compared to non-frail patients, only those frail patients who were over the age of 65 years were more likely to be admitted to a nursing home $(p<0.001)$. However, 30 -day readmissions were not significantly different according to the categorisation of frailty by the HFRS in different age groups (Table 2). Multilevel regression analysis suggested that frail patients as defined by the HFRS had a 1.7-fold increased risk of prolonged LOS (IRR 1.73, 95\% CI 1.65-1.82, $p$ value $<0.001$ ) and a 4-fold increased risk of predicting 30-day mortality (IRR 4.12, 95\% 2.71-6.27, $p<0.001$ ) after adjustment for age, sex, CCI, creatinine, BNP, troponin, CRP and albumin levels. However, HFRS was not found to predict 30-day readmissions after adjusted analysis (IRR 0.99, 95\% CI 0.96-1.03, $p$ value $=0.915$ ).

In this study, the ability to predict 30-day mortality in all hospitalised heart failure patients using age, sex, CCI and LACE score was good (c-statistic 0.74). Adding the HFRS to the above model improved its ability to predict 30-day mortality (c-statistic 0.80 ). Similarly, the model containing age, sex, CCI, LACE score and HFRS was a better predictor of prolonged LOS (LOS $>5$ days) (c-statistics 0.79 ) than the model without HFRS (c-statistic 0.74). However, the addition of the HFRS to the model containing age, sex, CCI and LACE did not improve the prediction for 30-day readmissions (c-statistic 0.57 without HFRS vs. 0.57 with HFRS). 
Table 2. Clinical outcomes according to frailty status.

\begin{tabular}{|c|c|c|c|c|}
\hline Outcome & $\begin{array}{c}\text { Low Risk of Frailty } \\
\text { HFRS }<5\end{array}$ & $\begin{array}{l}\text { Intermediate Risk of Frailty } \\
\text { HFRS 5-15 }\end{array}$ & $\begin{array}{l}\text { High Risk of Frailty } \\
\text { HFRS }>15\end{array}$ & $p$ Value \\
\hline & $n=2830$ & $n=817$ & $n=59$ & \\
\hline \multicolumn{5}{|l|}{ Overall } \\
\hline LOS median (IQR) & $4(2.5,6.7)$ & $7.4(4.7,11.8)$ & $14.5(8.6,20.6)$ & $<0.001$ \\
\hline $\begin{array}{c}\text { In hospital mortality } \\
n(\%)\end{array}$ & $98(3.5)$ & $118(14.4)$ & $22(37.3)$ & $<0.001$ \\
\hline 30-day mortality $n(\%)$ & $164(5.8)$ & $188(23.0)$ & $34(57.6)$ & $<0.001$ \\
\hline 180-day mortality $n(\%)$ & $390(13.8)$ & $299(36.6)$ & $42(71.2)$ & $<0.001$ \\
\hline 30-days readmissions $n(\%)$ & 545 (19.3) & 147 (17.9) & $15(25.4)$ & 0.329 \\
\hline NH placement $n(\%)$ & $137(4.8)$ & $83(10.2)$ & $9(15.3)$ & $<0.001$ \\
\hline \multicolumn{5}{|l|}{$<65$ years } \\
\hline LOS median (IQR) & $4.6(2.8,6.9)$ & $9.8(5.6,14.2)$ & $22.0(20.6,22.5)$ & $<0.001$ \\
\hline In hospital mortality $n(\%)$ & $8(1.3)$ & $9(8.9)$ & 0 & $<0.001$ \\
\hline 30-day mortality $n(\%)$ & $14(2.3)$ & $11(10.9)$ & $1(20.0)$ & $<0.001$ \\
\hline 180-day mortality $n(\%)$ & $36(6.0)$ & $20(19.8)$ & $2(40.0)$ & $<0.001$ \\
\hline 30-days readmissions $n(\%)$ & $99(16.6)$ & $20(19.8)$ & $1(20.0)$ & 0.713 \\
\hline NH placement $n(\%)$ & $2(0.3)$ & 0 & 0 & 0.837 \\
\hline \multicolumn{5}{|l|}{$65-89$ years } \\
\hline LOS median (IQR) & $4.0(2.5,6.7)$ & $7.8(5.1,13.2)$ & $15.2(14,17.6)$ & $<0.001$ \\
\hline In hospital mortality $n(\%)$ & $20(2.42)$ & $31(14.2)$ & $4(44.4)$ & $<0.001$ \\
\hline 30-day mortality $n(\%)$ & $34(3.8)$ & $40(18.3)$ & $6(66.7)$ & $<0.001$ \\
\hline 180-day mortality $n(\%)$ & $93(10.3)$ & $61(27.9)$ & $7(77.8)$ & $<0.001$ \\
\hline 30-days readmissions $n(\%)$ & $152(16.8)$ & $32(14.6)$ & $2(22.2)$ & 0.650 \\
\hline NH placement $n(\%)$ & $12(1.3)$ & $8(3.6)$ & $1(11.1)$ & 0.009 \\
\hline$\geq 80$ years & $3.9(2.4,6.4)$ & $6.9(4.2,10.7)$ & $13.4(6.2,19.1)$ & $<0.001$ \\
\hline \multicolumn{5}{|l|}{ LOS } \\
\hline In hospital mortality $n(\%)$ & $70(5.3)$ & $78(15.7)$ & $18(40.0)$ & $<0.001$ \\
\hline 30-day mortality $n(\%)$ & $116(8.7)$ & $137(27.6)$ & $27(60.0)$ & $<0.001$ \\
\hline 180-day mortality $n(\%)$ & $261(19.6)$ & $218(43.9)$ & $33(73.3)$ & $<0.001$ \\
\hline 30-days readmissions $n(\%)$ & $294(22.1)$ & $95(19.1)$ & $12(26.7)$ & 0.262 \\
\hline NH placement $n(\%)$ & $123(9.3)$ & $75(15.1)$ & $8(17.8)$ & 0.001 \\
\hline
\end{tabular}

HFRS, Hospital Frailty Risk Score; LOS, length of hospital stay; IQR, interquartile range; NH, nursing home.

Overall, 3110 (83.9\%) patients received one or more heart failure specific medications (beta blockers, ACEi/ARB and MRA). However, heart failure specific medications were less likely to be prescribed to frail patients when compared to the non-frail group $(77.4 \%$ vs. $85.9 \%, p$ value $<0.001)$. Patients who were categorised as non-frail according to the HFRS were more likely to receive beta blockers, ACE inhibitors and MRAs than those who were in the frail group ( $p$ value $<0.001$ ) (Table 1). Similarly, a significantly higher proportion of patients who were non-frail received aspirin, anticoagulants and statins when compared to non-frail patients ( $p$ value $<0.001$ ). However, there was no difference in the use of ARBs and digoxin between the frail and non-frail patients (Table 1). Both the HFRS and age were found to be negatively correlated with the use of heart failure targeted pharmacotherapy, although the correlation was weak (Spearman correlation coefficients -0.09 vs. -0.16 , both $p$ values $<0.001)$. Nevertheless, there was no correlation between Charlson index and use of heart failure specific pharmacotherapy (Spearman correlation coefficients $(-0.02$, $p$ value $=0.467)$. Over the duration of this study, the proportion of patients who received heart failure specific therapy decreased from $86.7 \%$ in 2015 to $82.9 \%$ in 2019 ( $p$ value $=0.03$ ) (Figure 4). However, although not reaching statistical significance, this trend was more apparent among frail patients (86\% in $2015 \mathrm{vs.76} \%$ in $2019, p=0.173$ ) when compared to non-frail patients $(87 \%$ in 2015 vs. $86 \%$ in $2019, p$ value $=0.247)$. 


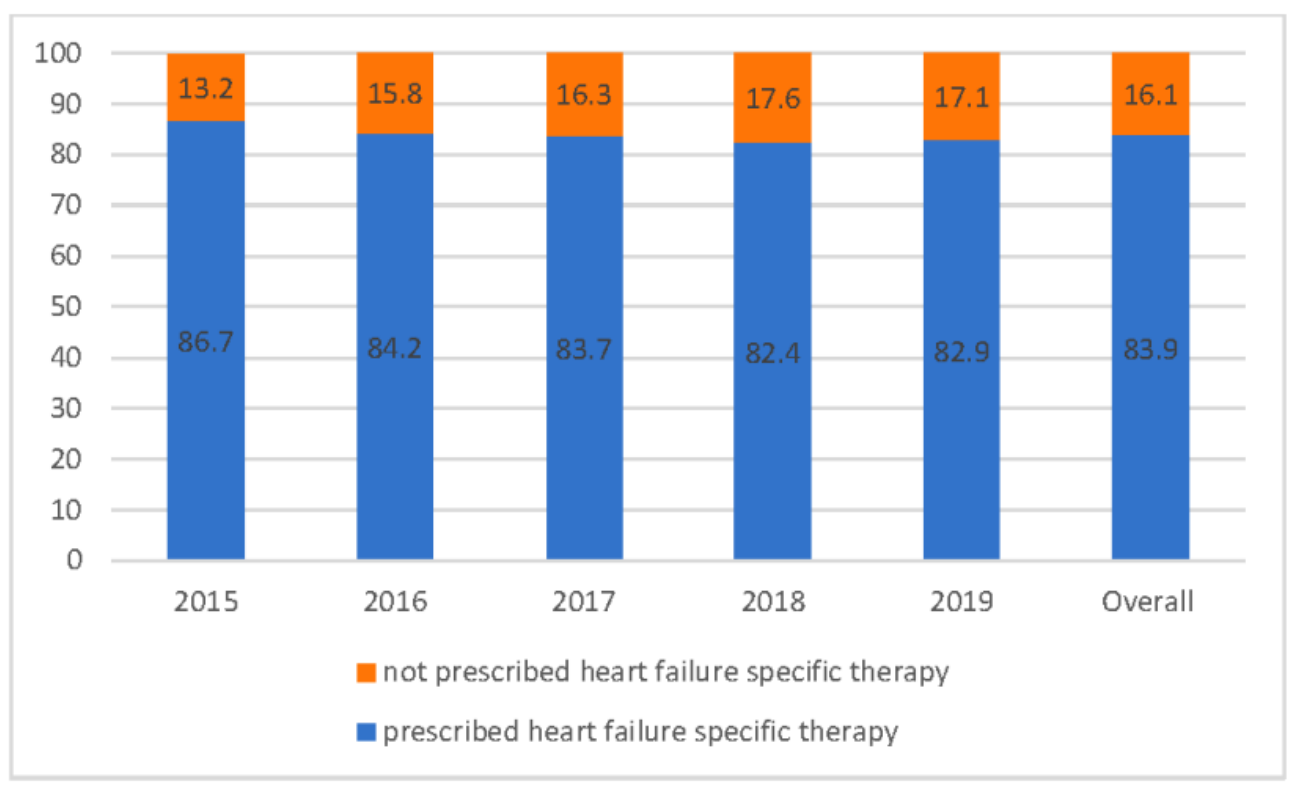

Figure 4. Trends in prescription of heart failure specific medications from 2015-2019.

\section{Discussion}

This study proved that, over a wide age range, a significant proportion of patients who were admitted with heart failure were identified as frail according to the HFRS and the proportion of inpatients with heart failure who were frail has increased in the last five years despite no significant aging of this population. Frailty as identified by the HFRS was only modestly correlated with age and CCI. HFRS predicted LOS, mortality and admissions to nursing home but not 30-day readmissions and the addition of HFRS increased prediction of clinical outcomes such as LOS and death above and beyond age, sex, CCI and LACE score. Frail heart failure patients were less likely to receive heart failure specific therapy when compared to non-frail patients with a trend towards decreased rate of prescription over the five years of the study period.

Although increasing HFRS scores were positively correlated with age and CCI, these associations were relatively weak. HFRS does reflect aspects of frailty, which are not captured by age and CCI. This is not surprising because HFRS is based on primary and secondary diagnostic codes, which reflect conditions such as falls, sarcopenia, incontinence and urinary tract infections; none of which is captured by the CCI [6].

Our study also shows that the addition of the HFRS to standard risk prediction models [2] for post discharge outcomes provided modest additional information in terms of predicting LOS and mortality but not readmissions. These findings are similar to a Canadian study [3], which included 26,266 hospitalised heart failure patients with a mean age of 77.4 years and found that addition of the HFRS only poorly predicted outcomes in the first 30-days after discharge from hospital. Similarly, an English Longitudinal Study on Ageing (ELSA) [17], which included 5294 patients over the age of 60 years, also found that the addition of frailty scores provided only marginal improvements in predicting clinical outcomes above chronological age for several different long-term outcomes. This may be because the HFRS is a cumulative deficit model of frailty and does not capture functional status and does not correlate with measures such as hand grip strength and gait speedwhich are typical phenotypic manifestations of frailty [18]. In addition, HFRS does not capture transient fluctuations induced by deconditioning associated with an acute hospital admission and also does not include variables, such as social support and socioeconomic status, which can influence outcomes such as unplanned hospital readmissions.

This study found that although there was a small change in age, the proportion of frail hospitalised heart failure patients increased between 2015 and 2019. This increment in frailty over time, although small, was statistically significant only in older patients 
( $\geq 65$ years). This could be related to an increase in the non-cardiovascular comorbidity burden of heart failure patients as has been reported by a number of recent studies $[19,20]$. This study also found that a significant proportion (15.1\%) of patients younger than 65 years of age met the HFRS case definition for frailty and, similar to the older counterparts, frailty was associated with worse outcomes in the younger age group. These results are similar to a study by McAlister et al. [3], who also found that up to a quarter of hospitalised heart failure patients under the age of 65 years may be frail. This finding indicates that frailty should be considered in younger patients admitted with heart failure and not just in older patients.

This study also found that frail patients are less likely to receive evidence-based heart failure specific therapy than non-frail patients and, over time, this disparity worsened. This trend over time was evident even in older patients who were classified as non-frail by the HFRS. Our data indicate that that there is only a weak correlation between the HFRS defined frailty and age and both HFRS and age are negatively correlated with the prescription of heart failure specific medications. These findings echo results of a recent Canadian study [3], which also found a weak correlation between HFRS and age $(r=0.20$, $p<0.001)$ and also found that both HFRS and age were negatively correlated with the prescription of heart failure specific therapies $(\mathrm{r}=-0.14$ and $\mathrm{r}=-0.23$, respectively, $p<0.05)$. It is possible that, in the absence of an objective assessment of frailty, clinicians rely on age as a proxy for frailty, so as to determine their potential suitability for receipt of heart failure specific pharmacotherapy, an assumption which has been proven to be wrong according to our study as well as others [3]. Since frail patients are at a high risk of adverse health outcomes, such as death or readmission [21,22], the potential absolute benefits of treatment may be greatest in this subgroup of patients [23].

Current guidelines from the European Society of Cardiology (ESC) and Heart Failure Association (HFA) [24] recommend heart failure specific pharmacotherapy irrespective of age. However, our study found that a large proportion of frail patients received suboptimal heart failure therapy. In fact, recommended heart failure therapy was less likely to be prescribed to patients who had a greater severity of frailty. The potential reasons could be a real or perceived greater likelihood of side-effects or intolerance to medications in frail patients. The other reason for physicians' inertia in prescribing medications in older frail patients could be due to a paucity of evidence because older patients have been systematically excluded from heart failure trials. This is evident from a recent study [22], which highlighted that after applying inclusion and exclusion criteria for frailty in the PARADIGM-HF, $83 \%$ of frail patients would have been excluded compared to $65 \%$ of nonfrail patients $(p<0.001)$. Similarly, in the recent DAPA-HF trial [25], which investigated the benefits of Dapagliflozin in heart failure, only a minority of patients $(24 \%)$ were $\geq 75$ years and this trial also excluded patients with severe chronic kidney disease (CKD) (GFR $<30 \mathrm{~mL} / \mathrm{min} / 1.73 \mathrm{~m}^{2}$ ) and the presence of comorbidities, which could limit life expectancy. These findings suggest that it is difficult for clinicians to ascertain the benefits of heart failure specific therapy in older frail subjects.

\section{Limitations}

We were unable to procure echocardiogram results of our patients and hence were unable to distinguish the impact of frailty in patients with heart failure with reduced and preserved ejection fractions. Nonetheless, the severity of heart failure in these patients was judged from the BNP levels [26]. We have no data on the clinical presentation of these patients nor on the presence of any contraindications to evidence-based heart failure medications and thus cannot comment upon the appropriateness of therapy. Due to the observational design of this study and the possibility of residual confounding, we cannot conclusively state the underlying reasons for the observed associations. Finally, we cannot rule out any change in the depth of coding over years in our hospitals because it has been found that hospitals with better depth of coding had a higher proportion of patients meeting the HFRS case definition of frailty. 


\section{Conclusions}

This study has found that HFRS defined frailty has increased in the last five years in hospitalised heart failure patients in Australia and HFRS reflects aspects of frailty not determined by age or Charlson index. The addition of HFRS to standard risk prediction models comprising of age and Charlson provides only marginal improvements in the prediction of clinical outcomes. The prescription of heart failure targeted therapy remains poor, especially in frail hospitalised patients, and has deteriorated in the last five years. Future trials in heart failure patients should include older frail patients and the assessment of frailty should be routinely included in the management of heart failure patients because frail patients, irrespective of their age, have poorer outcomes and may gain greater benefits from heart failure targeted therapies than non-frail patients.

Author Contributions: Conceptualisation, Y.S. and C.T.; methodology, Y.S.; software, Y.S., C.H. and P.H.; validation, Y.S. and C.H.; formal Analysis, Y.S. and C.H.; investigation, C.H., C.T. and Y.S.; resources, Y.S.; data curation, P.H. and C.H.; writing—original draft preparation, Y.S.; writingreview and editing, Y.S. and C.T.; visualization, Y.S.; supervision, Y.S. and C.T.; project administration, Y.S. All authors have read and agreed to the published version of the manuscript.

Funding: This research received no external funding.

Institutional Review Board Statement: The study was conducted according to the guidelines of the Declaration of Helsinki and approved by the Southern Adelaide Human Clinical Research Ethics Committee (protocol code 3.19, date of approval 4 November 2019).

Informed Consent Statement: Informed consent was not obtained due to retrospective collection of data in this study.

Data Availability Statement: The data presented in this study are available on request from the corresponding author only after permission is granted by the ethics committee.

Conflicts of Interest: The authors declare no conflict of interest.

\section{References}

1. Zhang, Y.; Yuan, M.; Gong, M.; Tse, G.; Li, G.; Liu, T. Frailty and Clinical Outcomes in Heart Failure: A Systematic Review and Meta-analysis. J. Am. Med. Dir. Assoc. 2018, 19, 1003-1008.e1. [CrossRef]

2. Zhou, H.; Della, P.R.; Roberts, P.; Goh, L.; Dhaliwal, S.S. Utility of models to predict 28-day or 30-day unplanned hospital readmissions: An updated systematic review. BMJ Open 2016, 6, e011060. [CrossRef]

3. McAlister, F.A.; Savu, A.; Ezekowitz, J.A.; Armstrong, P.W.; Kaul, P. The hospital frailty risk score in patients with heart failure is strongly associated with outcomes but less so with pharmacotherapy. J. Intern. Med. 2020, 287, 322-332. [CrossRef]

4. Tanaka, S.; Kamiya, K.; Hamazaki, N.; Matsuzawa, R.; Nozaki, K.; Nakamura, T.; Yamashita, M.; Maekawa, E.; Noda, C.; Yamaoka-Tojo, M.; et al. Short-Term Change in Gait Speed and Clinical Outcomes in Older Patients With Acute Heart Failure. Circ. J. 2019, 83, 1860-1867. [CrossRef] [PubMed]

5. Sillars, A.; Celis-Morales, C.A.; Ho, F.K.; Petermann, F.; Welsh, P.; Iliodromiti, S.; Ferguson, L.D.; Lyall, D.M.; Anderson, J.; Mackay, D.F.; et al. Association of Fitness and Grip Strength With Heart Failure: Findings From the UK Biobank Population-Based Study. Mayo Clin. Proc. 2019, 94, 2230-2240. [CrossRef] [PubMed]

6. Gilbert, T.; Neuburger, J.; Kraindler, J.; Keeble, E.; Smith, P.; Ariti, C.; Arora, S.; Street, A.; Parker, S.; Roberts, H.C.; et al. Development and validation of a Hospital Frailty Risk Score focusing on older people in acute care settings using electronic hospital records: An observational study. Lancet 2018, 391, 1775-1782. [CrossRef]

7. McAlister, F.; van Walraven, C. External validation of the Hospital Frailty Risk Score and comparison with the Hospital-patient One-year Mortality Risk Score to predict outcomes in elderly hospitalised patients: A retrospective cohort study. BMJ Qual. Saf. 2018, 28, 284-288. [CrossRef] [PubMed]

8. Eckart, A.; Hauser, S.I.; Haubitz, S.; Struja, T.; Kutz, A.; Koch, D.; Neeser, O.; Meier, M.A.; Mueller, B.; Schuetz, P. Validation of the hospital frailty risk score in a tertiary care hospital in Switzerland: Results of a prospective, observational study. BMJ Open 2019, 9, e026923. [CrossRef] [PubMed]

9. Greene, S.J.; Butler, J; Albert, N.M.; DeVore, A.D.; Sharma, P.P.; Duffy, C.I.; Hill, C.L.; McCague, K.; Mi, X.; Patterson, J.H.; et al. Medical Therapy for Heart Failure with Reduced Ejection Fraction: The CHAMP-HF Registry. J. Am. Coll. Cardiol. 2018, 72, 351-366. [CrossRef]

10. Brunner-La Rocca, H.P.; Linssen, G.C.; Smeele, F.J.; van Drimmelen, A.A.; Schaafsma, H.J.; Westendorp, P.H.; Rademaker, P.C.; van de Kamp, H.J.; Hoes, A.W.; Brugts, J.J. Contemporary Drug Treatment of Chronic Heart Failure With Reduced Ejection Fraction: The CHECK-HF Registry. JACC Heart Fail. 2019, 7, 13-21. [CrossRef] [PubMed] 
11. Bhatt, A.S.; DeVore, A.D.; DeWald, T.A.; Swedberg, K.; Mentz, R.J. Achieving a Maximally Tolerated $\beta$-Blocker Dose in Heart Failure Patients: Is There Room for Improvement? J. Am. Coll. Cardiol. 2017, 69, 2542-2550. [CrossRef] [PubMed]

12. Allen, L.A.; Fang, J.C. Goldilocks Dilemma of Dose Titration in Heart Failure With Reduced Ejection Fraction: Too Little, Too Much, or Just Right? Circ. Heart Fail. 2017, 10, e004406. [CrossRef] [PubMed]

13. Samsky, M.D.; Ambrosy, A.P.; Youngson, E.; Liang, L.; Kaul, P.; Hernandez, A.F.; Peterson, E.D.; McAlister, F.A. Trends in Readmissions and Length of Stay for Patients Hospitalized With Heart Failure in Canada and the United States. JAMA Cardiol. 2019, 4, 444-453. [CrossRef] [PubMed]

14. Joshy, G.; Korda, R.J.; Abhayaratna, W.P.; Soga, K.; Banks, E. Categorising major cardiovascular disease hospitalisations from routinely collected data. Public Health Res. Pract. 2015, 25, e2531532. [CrossRef] [PubMed]

15. Jelizarow, M.; Mansmann, U.; Goeman, J.J. A Cochran-Armitage-type and a score-free global test for multivariate ordinal data. Stat. Med. 2016, 35, 2754-2769. [CrossRef]

16. Yazdan-Ashoori, P.; Lee, S.F.; Ibrahim, Q.; Van Spall, H.G. Utility of the LACE index at the bedside in predicting 30-day readmission or death in patients hospitalized with heart failure. Am. Heart J. 2016, 179, 51-58. [CrossRef]

17. Aguayo, G.A.; Vaillant, M.T.; Donneau, A.F.; Schritz, A.; Stranges, S.; Malisoux, L.; Chioti, A.; Guillaume, M.; Muller, M.; Witte, D.R. Comparative analysis of the association between 35 frailty scores and cardiovascular events, cancer, and total mortality in an elderly general population in England: An observational study. PLoS Med. 2018, 15, e1002543. [CrossRef]

18. Kua, J.; Ramason, R.; Rajamoney, G.; Chong, M.S. Which frailty measure is a good predictor of early post-operative complications in elderly hip fracture patients? Arch. Orthop. Trauma Surg. 2016, 136, 639-647. [CrossRef]

19. Sharma, A.; Zhao, X.; Hammill, B.G.; Hernandez, A.F.; Fonarow, G.C.; Felker, G.M.; Yancy, C.W.; Heidenreich, P.A.; Ezekowitz, J.A.; DeVore, A.D. Trends in Noncardiovascular Comorbidities Among Patients Hospitalized for Heart Failure: Insights From the Get With The Guidelines-Heart Failure Registry. Circ. Heart Fail. 2018, 11, e004646. [CrossRef]

20. Iorio, A.; Senni, M.; Barbati, G.; Greene, S.J.; Poli, S.; Zambon, E.; Di Nora, C.; Cioffi, G.; Tarantini, L.; Gavazzi, A.; et al. Prevalence and prognostic impact of non-cardiac co-morbidities in heart failure outpatients with preserved and reduced ejection fraction: A community-based study. Eur. J. Heart Fail. 2018, 20, 1257-1266. [CrossRef]

21. Stillman, G.R.; Stillman, A.N.; Beecher, M.S. Frailty Is Associated With Early Hospital Readmission in Older Medical Patients. J. Appl. Gerontol. 2021, 40, 38-46. [CrossRef] [PubMed]

22. Sze, S.; Pellicori, P.; Zhang, J.; Weston, J.; Squire, I.B.; Clark, A.L. Effect of frailty on treatment, hospitalisation and death in patients with chronic heart failure. Clin. Res. Cardiol. 2021, 110, 1249-1258. [CrossRef] [PubMed]

23. McAlister, F.A. Applying evidence to patient care: From black and white to shades of grey. Ann. Intern. Med. 2003, 138, 938-939. [CrossRef]

24. Gillespie, N.D.; McMurdo, M.E.; Struthers, A.D. Diagnosis and management of heart failure: Implications of the recent European Society of Cardiology Guidelines for the older patient. Age Ageing 2003, 32, 563-565. [CrossRef]

25. McMurray, J.J.V.; DeMets, D.L.; Inzucchi, S.E.; Køber, L.; Kosiborod, M.N.; Langkilde, A.M.; Martinez, F.A.; Bengtsson, O.; Ponikowski, P.; Sabatine, M.S.; et al. The Dapagliflozin And Prevention of Adverse-outcomes in Heart Failure (DAPA-HF) trial: Baseline characteristics. Eur. J. Heart Fail. 2019, 21, 1402-1411. [CrossRef]

26. Mueller, C.; McDonald, K.; de Boer, R.A.; Maisel, A.; Cleland, J.G.F.; Kozhuharov, N.; Coats, A.J.S.; Metra, M.; Mebazaa, A.; Ruschitzka, F; et al. Heart Failure Association of the European Society of Cardiology practical guidance on the use of natriuretic peptide concentrations. Eur. J. Heart Fail. 2019, 21, 715-731. [CrossRef] [PubMed] 